\title{
Some Fundamental Aspects of Mechanics of Nanocomposite Materials and Structural Members
}

\author{
Alexander N. Guz and Jeremiah J. Rushchitsky \\ S. P. Timoshenko Institute of Mechanics, Nesterov Street 3, 03680 Kiev, Ukraine \\ Correspondence should be addressed to Jeremiah J. Rushchitsky; rushch@inmech.kiev.ua
}

Received 24 June 2013; Accepted 6 September 2013

Academic Editor: Ping Xu

Copyright (C) 2013 A. N. Guz and J. J. Rushchitsky. This is an open access article distributed under the Creative Commons Attribution License, which permits unrestricted use, distribution, and reproduction in any medium, provided the original work is properly cited.

\begin{abstract}
This paper is devoted to formulation and analysis of fundamental aspects of mechanics of nanocomposite materials and structural members. These aspects most likely do not exhaust all of the possible fundamental characteristics of mechanics of nanocomposite materials and structural members, but, nevertheless, they permit to form the skeleton of direction of mechanics in hand. The proposed nine aspects are described and commented briefly.
\end{abstract}

\section{Introduction}

The specificity of mechanics as science consists in that it is one of the most important sciences of fundamental character and at the same time its urgency is defined by significance for engineering of many problems of mechanics. At all of the stages of human progress, starting with the ancient world, the importance of mechanics for engineering cannot be overemphasized: in many cases, mechanics, and engineering were considered as a single whole. This specificity of mechanics is shown up, when the mechanics of materials was formed as the scientific direction, in which uniting of mechanics and engineering is very significant.

Mechanics of materials as the direction has been clearly formed in the last century within the framework of solid mechanics along with mechanics of structural members, when the investigations related to the development of new materials were essentially extended. Actually, the sufficiently ponderable part of investigations, which are carried out on solid mechanics, is represented in mechanics of materials. This situation resulted in that in some universities solid mechanics is included in different courses on material science.

On the whole, investigations on mechanics of materials are defined or characterized by the fact that the information on internal structure of material is always taken into account, although in different extent. In the most part of investigations on mechanics of materials, this information is used for characterization or identification of materials. In this case, the internal structure is considered in analysis of photo of internal structure and its change under force and technological actions only. In the slightly less part of investigations on mechanics of materials, information on internal structure is included in the models of materials and is utilized in the statement and solution of corresponding problems of mechanics of materials. This allows to separate the structural mechanics of materials as the independent scientific direction within the framework of mechanics of materials $[1,2]$.

Thus, the structural mechanics of materials is meant to be the part of investigations on mechanics of materials, in which the internal structure of materials is taken into account in quantitative and qualitative sense, when the models of materials are being constructed and the corresponding problems are being studied.

When structural mechanics of materials is defined in such a way, then the object of its study is the large class of modern materials, including reinforced concrete, internal structures of which is defined by presence of armature; metals, alloys, and ceramics, internal structure of which are defined by presence of grains and other structural components; composite materials, internal structures of which are defined by presence of granules, fibers, and layers; and nanocomposites, internal structures of which are defined by presence of nanogranules, nanofibers, and nanolayers. 
In this paper, "macromechanics, mesomechanics, micromechanics, and nanomechanics of materials are the component parts of structural mechanics of materials [1-3]."

The corresponding notions and definitions to the four component parts mentioned above are comparatively established and widely used. The one only and necessary common requirement for all four scientific directions is to take into account the internal structure of material in mechanical models and in solving the corresponding problems.

Below, the proposed fundamental aspects of mechanics of nanocomposite materials and structural members permit to form the skeleton of this direction of mechanics. Introducing the term structural members has the goal of considering the following nanocomposite materials object of mechanics, because this object is prevailing in engineering. But relating the structural composite elements to the ones of nanolevels imports the additional restrictions on analysis within the framework of nanomechanics. Furthermore, the above-mentioned aspects are considered sequentially.

\section{Aspect 1: Analysis of Internal Structure and Structural Levels}

An analysis of internal structure in materials and usage of the notion of structural levels give the straight track to differing the nanomechanics from macro-, meso-, and micromechanics. This notion arose in micromechanics, but it became very productive and maybe the most important for description of nanomechanics, too.

To characterize quantitatively the internal structure of materials as objects of study in structural mechanics of materials, it is expedient to introduce the geometrical parameter $h$.

(i) In the case of reinforced concrete, the parameter $h$ characterizes the mean value of minimal diameters of cross-section of metallic armature.

(ii) In the case of metals, alloys, and ceramics, the parameter $h$ characterizes the mean value of minimal sizes of cells, grains, and other structural inhomogeneities.

(iii) In the case of composite materials with polymeric and metallic matrices, the parameter $h$ characterizes the mean value of minimal diameters of grains for materials of granular structure, the mean value of minimal diameters of cross-sections of fibers for fibrous materials, and the mean value of minimal thickness of layers of components for layered materials.

(iv) In the case of nanomaterials (nanocomposites), the parameter $h$ characterizes the mean value of minimal diameters of nanoparticles or nanolayers.

It seemed to be appropriate to propose the limits of changing the parameter $h$, which determine relating the corresponding materials to macromechanics, mesomechanics, micromechanics, or nanomechanics. It is necessary to note that some scientists proposed variants of changing the parameter $h$-levels of changing of $h$. As a consequence of analysis

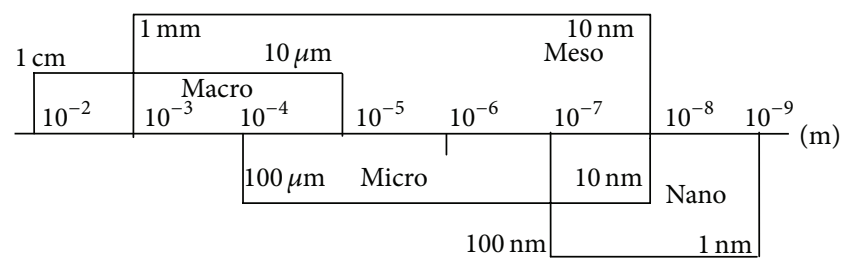

FIGURE 1: Structural levels.

of proposals of different authors, the next four levels for parameter $h$ were proposed in publications $[4,5]$ :

$$
\begin{aligned}
& \text { macro, } 10^{-2} \mathrm{~m} \geq h \geq 10^{-5} \mathrm{~m} ; \quad \text { meso, } 10^{-5} \mathrm{~m} \geq h \geq 10^{-7} \mathrm{~m} ; \\
& \text { micro, } 10^{-7} \mathrm{~m} \geq h \geq 10^{-8} \mathrm{~m} ; \quad \text { nano, } 10^{-8} \mathrm{~m} \geq h \geq 10^{-9} \mathrm{~m} .
\end{aligned}
$$

Let us note that the atom level (distance between atom planes in crystal lattice) has the order of one or more angstroms $\left(10^{-10} \mathrm{~m}\right)$; therefore, the nanolevel in (1) is conditionally bounded by $10^{-9} \mathrm{~m}$.

For the convenience of readers, the relationships between used length units are shown as follows:

$$
\begin{gathered}
\text { meter, } \mathrm{m} \text {; centimeter, } \mathrm{cm}=10^{-2} \mathrm{~m} ; \\
\text { millimeter, } \mathrm{mm}=10^{-3} \mathrm{~m} ; \text { micron, } \mu \mathrm{m}=10^{-6} \mathrm{~m} ; \\
\text { nanometer, } \mathrm{nm}=10^{-9} \mathrm{~m} \text {; angstrom, } \AA=10^{-10} \mathrm{~m} .
\end{gathered}
$$

Attention on that proposed in (1) four levels for parameter $h$ do not overlap one another. In development of some scientific directions related to macro-, meso-, micro- and nanomechanics, the investigations are carryied out in the framework of scale levels, which overlap one another that is, the four level are considered in the extended interpretation.

Based on analysis of proposals of different authors, within the above mentioned extended interpretation the four levels for parameter $h$ were proposed in publications $[4,5]$

$$
\begin{aligned}
& \text { macro, } 10^{-2} \mathrm{~m} \geq h \geq 10^{-5} \mathrm{~m} ; \quad \text { meso, } 10^{-3} \mathrm{~m} \geq h \geq 10^{-8} \mathrm{~m} ; \\
& \text { micro, } 10^{-4} \mathrm{~m} \geq h \geq 10^{-8} \mathrm{~m} ; \quad \text { nano, } 10^{-7} \mathrm{~m} \geq h \geq 10^{-9} \mathrm{~m} .
\end{aligned}
$$

Schematically, four levels (3) are shown in Figure $1[1,2,4,5]$.

Thus, within the framework of this aspect, the tool of identification of the material as the nanomaterial is generated.

\section{Aspect 2: Modeling in Mechanics of Nanocomposite Materials and Structural Members}

Constructing the mechanical models can be thought as the main goal of mechanics. Let us remember the well-known sentence of Truesdell's [6]: in fact, mechanics is an infinite class of models to represent certain aspects of nature. Mechanics of materials, of course, is understood as part of mechanics 
and can be meant as exploring the infinite set of mechanical models of materials. Usually, the mechanical models are related to the theoretical part of mechanics and are formulated in terms of mathematics and physics. Nowadays, the theoretical models consist of the structural and mathematical parts, but, traditionally, they are meant as the mathematical models. Each theoretical model in mechanics underlies, as a rule, the corresponding mechanical theory.

The models of materials are by their sense some idealizations of the real materials, and applicability of every model should be tested. Thus, the experimental mechanics presents the special part of mechanics and forms the fundamental knowledge, which arises owing to direct contact with real nature (in solid mechanics, with real materials). For fundamental sciences, the necessity of attention to experiments and practice had been formulated far back by Leibniz in his statement "theoria cum practica." Today, it is understood as the necessity for any theory to amplify with experimentations. 200 years later, Boltzmann stated "nothing is so practical as the theory." In 1926, in a talk between Werner von Heisenberg and Albert Einstein, Heisenberg stated that each theory, in its building, must correspond to only those observed by this time facts. Einstein answered that it could be wrong to try to build the theory only on observed facts. Really, it happens the vice versa. Theory determines, what we can observe.

So, when the process of deformation of materials is being described (modeled), then different models are applied taking into account the discrete structure of material at the atom level and not taking into account this structure within the framework of continuum representation.

Note that the continuum representation of material consists in that real piece of material (material body) is replaced by continuum of the same geometrical shape. In each point of continuum, the values of physical-mechanical parameters of material (physical-mechanical properties) and physicalmechanical fields (stresses, strains, temperature, and so on) are considered.

The following three types of media are studied:

(i) homogeneous media (continua), when values of physical-mechanical parameters (constants) do not depend on the point (they are one and the same for all of the medium);

(ii) piecewise homogeneous media (continua), consisting of separate parts of homogeneous media, which are continuously jointed and form one continuum;

(iii) inhomogeneous media (continua) with continuously changing inhomogeneity.

The main advantage of continuum description consists in that it permits to apply the methods of continuous mathematics and, in particular, the differential and integral calculi. At present, owing to active development of the finite element method and discrete mathematics, the significance of continuum representation will be possibly refined.

Among the above mentioned models, the following principally distinguished models should be marked out as such, which are the most widespread ones.
Model 1: the model of discretely arranged rigid particles (which model the atoms), interacting with themselves.

Model 2: the model of piece-wise continuous body, each component of which is described within the framework of continuum representation.

Model 3: the model of homogeneous body, which is analyzed within the framework of continuum representation.

In solid physics, the investigations are carried out within the framework of crystal lattice concept, that is, within the framework of model 1, in which interaction among atoms is put into effect by interatomic interaction forces caused by potentials of different structure. Such approach is represented in a number of monographs, among which it seems that the quite actively cited monograph [7] should be pointed, and in plentiful publications in scientific journals.

In particular, the model exists for discretely arranged rigid particles (balls), placed at nodes of crystal lattice and jointed by springs. In this case, the interaction among neighbouring balls is realized by the link of their springs. This model is used in mechanics, too. As an example, the monograph [8] can be pointed out, in which this model is applied to study dynamical processes occurring in the fracture wave.

In micromechanics of composite materials, the investigations are carried out within the framework of model 2the model of piece-wise homogeneous body, consisting of separate particles of the filler (reinforcing elements) and the binder (matrix). Description of deformation of each particle of the filler and the matrix is done within the framework of continuum representation. In this model, the interaction among separate particles of the filler is realized through the continuous body (matrix) by means of condition of continuity of stress and displacement vectors at the fillerbinder interface. On the interface, the different variants of joining the filler and the binder can occur in some materials, which is reflected in changing the concrete form of conditions of continuity. Such an approach is represented in a row of monograph editions ([9-20] and others) and in the large number of journal publications (e.g., [21, 22]).

Model 3-the model of homogeneous body with averaged properties-is successfully used in mechanics of composite materials. At that, the body is assumed frequently to be anisotropic. Then, in the most part of cases, composites are assumed to be orthotropic ones. The same model is used in mechanics of structural elements (shells, plates, rods, and so on) made of composite materials. It is described in many monographs and original papers [23-49]. Usually, in macroand mesomechanics, only models 2 and 3 are used, since on this scale level the components of composite material are the sufficient big pieces of real material. The problem of discrete structure of the piece is already not actual. It is worthy to note that for model 2 the homogenization can be required.

Undoubtedly, the essential part of investigations in mechanics of structural members is referring to the case when through the thickness the member consists of several homogeneous anisotropic layers. In these investigations, 
the two-dimensional (for shells and plates) and onedimensional (for rods) applied theories are mainly considered, which are constructed by introduction of hypotheses on distribution of stresses and displacements through the thickness.

Above, three models are considered comparatively briefly. These models along with other models are everywhere applied in structural mechanics of materials. Each of the models has its possibilities, advantages, disadvantages, when in mechanics of materials the concrete phenomena are being described, and specific difficulties in the realization.

Probably, the most complex model from the point of view of obtaining the concrete results is the second onethe model of piecewise homogeneous body. Let us show in support two reasons.

Reason 1 consists in that within the framework of this model each reinforcing element is studied within the framework of continuum mechanics-solid mechanics, which is developed very well.

Reason 2 consists in that interaction of different reinforcing elements occurs through the matrix, which is also studied within the framework of continuum mechanics-solid mechanics and permits therefore to use plenty of analytical approaches.

In mechanics of composite materials, three models discussed above as well as many other models are applied to composite materials in the form of the sequence (chain) of models, which provide at the final stage the study within the structural members made of these composite materials. This reflects the specificity of mechanics as science.

Obviously, the sequences (chains) of used models for different composite materials, and studied within them, phenomena are different too, because the level scales (1) and (3) and Figure 1 should be taken into account. So, for nanocomposites, models 1,2 , and 3 are included into the sequence, whereas for macro-, meso- and microcomposites, only models 2 and 3 are inculded.

When mechanics of composite materials are being constructed in the sense noted above, then different approaches and methods are utilized, which correspond to different scientific directions and scientific positions of single scientists. Nevertheless, despite such diversity of scientific directions, in construction of mechanics of composite materials, two basic principles (two basic concepts) are applied on principle of continualization and principle of homogenization, which are formulated below.

The principle of continualization consists in

\section{"that the discrete system is changed (modeled) by the continuous system (medium) and for the continuous system (medium) the corresponding averaged proper- ties are determined."}

This principle is used widely, for example, within the framework of model 1 in transition to the continuum theory of dislocations in crystal lattices.

The principle of homogenization consists in "changing (modeling) the piece-wise homogeneous system, deforming the elements (pieces) of which is described by the relationships of continuum mechanics of solids, by the homogeneous continuous system (medium), and by the determination of necessary averaged properties within the framework of the homogeneous continuous system (medium)."

The principle of homogenization is widely used within the framework of model 2 in micromechanics of composite materials, when different problems of statics, dynamics, stability, and fracture are being studied.

Usually, the area which a continuously inhomogeneous body (e.g., a composite material with the continuous changing in some direction number of micro- or nanospheres) or piece-wise homogeneous body (e.g., a composite material with uniformly distributed micro- or nanospheres by all of the directions) occupies is chosen, dimensions of which are essentially of less body sizes. This area should contain the sufficiently large number of inhomogeneities (e.g., granules) to provide the averaging correctness. Such an area is called the representative volume.

The averaged properties of the volume are usually attributed to the point at the volume center. As a result, the averaged properties are evaluated at every point of the body, and these properties should be constant-the body becomes the homogeneous one.

Very often, authors of different publications on materials are showing the color pictures of representative volumes in the form of cubes filled of discrete particles, which are looking very nice but do not image as a rule the real discrete structure.

The representative volume side length is compared with the characteristic length of body internal structure or with the characteristic length of inhomogeneities in the body (e.g., with micro- or nanosphere diameter). Exceeding the first length over the second one one order or more gives grounds to apply the averaging procedure.

Let us note that, from the abovestated continualization and homogenization principles, their principled distinction and methodological commonality follow (especially, in relation to the initial systems, to which they are applied).

Let us note finally that the procedures of continualization and homogenization are realized by means of different methods of averaging. At that, as a rule, the notion of representative volume is used.

Furthermore, three basic moments in realization of modeling with using the notion of representative volume and methods of averaging will be pointed.

Moment 1. When the notion of representative volume, which will be later denoted as $V_{\Pi}$, is being introduced, it is assumed that the minimal linear sizes of volume $V_{\Pi}$ are significantly more than the maximal sizes of discrete particles (in case of model 1) or maximal sizes of homogeneous parts of material (in case of model 2). Thus, the linear sizes of representative volume must be significantly more introduced before the geometrical parameter $h$.

Usually, the majority of authors are assuming that exceeding the first value over the second value on one or more orders gives grounds for the next modeling and averaging.

Parallel to selection of the representative volume $V_{\Pi}$ (which is separated in the initial model: model 1 or 2), select the equivalent volume $V_{E}$ also within the framework of 
the final model: the model of homogeneous body (model 3). Assume that volume $V_{\Pi}$ has the same shape as volume $V_{E}$ and is oriented in the same way. In this regard, the following denotation is used frequently:

$$
V_{\Pi}=V_{E}=V
$$

Moment 2. When the material is being modeled (the values and parameters in the equivalent volume $V_{E}$ being determined by means of averaging through corresponding values and parameters in the representative volume $V_{\Pi}$ ), it is assumed that the only homogeneous fields of stresses, strains, and so on are arising in the equivalent volume

$$
\left\langle\sigma_{i j}\right\rangle=\text { const }_{i j}, \quad\left\langle\varepsilon_{n m}\right\rangle=\text { const }_{n m}, \ldots
$$

It is expedient to stress that only in the analysis situations, corresponding to conditions (5), the possibility exists to determine the averaged values of different parameters (constants), which are included within the constitutive equations for homogeneous material (the model 3). At that, the methods of averaging are used for different quantities.

Consider as an example the procedure of determination of potential energy of deformation of elastic body in volumes $V_{\Pi}$ and $V_{E}$, for which corresponding denotations $E_{\Pi}$ and $E_{E}$ will be introduced. Also, denote by $\Phi\left(\varepsilon_{i j}\right)$ the specific potential energy of deformation of elastic material with internal structure (model 1 or 2), which is referred to as the unit of volume $V_{\Pi}$ and in which the components of the Green strain tensor according to model 2 or 3 are denoted as $\varepsilon_{i j}$. In his case, for volume $V_{\Pi}$, the following formula is valid:

$$
E_{\Pi}=\int_{V_{\Pi}} \Phi\left(\varepsilon_{i j}\right) d V_{\Pi}
$$

For volume $V_{E}$ (in which the stress and strain fields are already homogeneous), the energy can be evaluated too as following:

$$
E_{E}=\left(\int_{0}^{\left\langle\varepsilon_{i j}\right\rangle}\left\langle\sigma_{i j}\right\rangle d\left\langle\varepsilon_{i j}\right\rangle\right) V_{E}
$$

In the case of linearly elastic body, the following expression for volume $V_{E}$ can be obtained from formula (7) as follows:

$$
E_{E}=\left(\frac{1}{2}\right)\left(\left\langle\sigma_{i j}\right\rangle\left\langle\varepsilon_{i j}\right\rangle\right) V_{E}
$$

From (4) and (8), for the material with volume $V$ in different modeling from the initial model (model 1 or 2 in volume $V_{\Pi}$ ) to the final model (model 3 in volume $V_{E}$ ), when potential energy of elastic deformation is being averaged, the relationships can be written as

$$
\left\langle\sigma_{i j}\right\rangle\left\langle\varepsilon_{i j}\right\rangle=\frac{2}{V} \int_{V} \Phi\left(\varepsilon_{n m}\right) d V
$$

It is necessary to note that, along with expression (8), in carrying out the operation of averaging and evaluation of values of averaged parameters (constants), similar expressions are used, which follow from conditions of equilibrium for different structural components in $V_{\Pi}$ and from conditions of their common deformation.

Moment 3. The results of modeling and averaging, within the framework of approaches discussed in moments 1 and 2, are applicable in description of stress, strain, and other fields, which change insignificantly on distances of the same order as sizes of the representative volume $V_{\Pi}$.

If the stated, in description of moment 1 , reasons on determination of the linear sizes of the representative volume $V_{\Pi}$ through the introduced before geometrical parameter $h$ are taken into account, then the following conclusion can be achieved.

\section{"The results of modeling and averaging from above are applicable in description of stress, strain, and other fields which change insignificantly on distances exceeding one order or more the value of geometrical parameter $h$."}

Note that, in the practical realization of averaging procedure, the majority of authors are assuming additionally that in the material structural components (within the framework of the representative volume $V_{\Pi}$ ) the homogeneous fields of stresses, strains, and other quantities are realized (different for filler and binder). Such an approach simplifies essentially evaluation of integrals of the type of the right side of expression (8).

Thus, the shown description of aspect 2 can be related to arguments about similarity of all four parts of structural mechanics of materials, because all of the continuum mechanics models of these parts are identical.

\section{Aspect 3: Only Two Basic Models}

The feature of composite materials is their forming from the binder (matrix) and fillers (reinforcing elements). When composites as materials with the clearly shown internal structure are modeled, a row characterizing this structure geometrical parameters should be known.

Of course, when the approaches and methods of mechanics of composite materials of any level of internal structure are developed, one cannot orient the geometrical parameter $h$ only, because it determines a place of the composite in the hierarchical structure of materials only. It is necessary to take into account the character of mechanical processes under consideration.

In this regard, the geometrical parameter $L$ is introduced, which characterizes the variability of mechanical fields by spatial coordinates. Also, it turned out to be expedient to introduce for nanoformations the geometrical parameter $h^{*}$, which characterizes the mean value of distances between centers of particles in the internal structure of nanoformation. 
The introduced parameters $L, h$, and $h^{*}$ enable the determination of two essentially different models and corresponding methods of their analysis within the framework of mechanics of composite materials.

Model 1. This model is the piecewise homogeneous medium or model of discrete particles. It is applied when parameter $L$ is the quantity of the same order or less as compared with the parameters $h, h^{*}$ characterizing the structure of composite material. This condition can be represented by use of some inequalities as follows

$$
L \approx h, \quad L<h, \quad L \approx h^{*}, \quad L<h^{*} .
$$

If at least one of conditions (10) is fulfilled, then deformation of matrix and reinforcing elements is described by relationships of the continuum solid mechanics under some conditions at interfaces, which correspond to conditions of continuity of stress and displacement vectors.

When the 3D relationships of continuum solid mechanics are used, this approach is the most exact and rigorous within the framework of continuum solid mechanics. Using this model, the investigations of problems of statics, dynamics, stability, and fracture in mechanics of composite materials are carried out. If at least one of conditions (10) is fulfilled when applied to a nanoformation, then the motion of this nanoformation is described by relationships of the model of discrete particles.

Model 2. This model is the piecewise homogeneous anisotropic body with averaged properties. It is utilized when the characterizing of a variability of mechanical fields by the spatial variables parameter $L$ which is the quantity more essentially than the characterizing of the structure of composite materials parameters $h, h^{*}$. This condition can be written in the form of inequalities

$$
L \gg h, \quad L \gg h^{*} .
$$

In this case, a composite material is modeled by the homogeneous anisotropic body with averaged properties. Some intermediate criteria between (10) and (11) can be formulated, but they will be of less importance when comparing with (10) and (11). For example, in the case of pure filling (very small volume fraction of fillers), the mean value of minimal diameters of fillers $h$ is essentially smaller than the value of distance among the filler centers $h^{*}$; that is, $h \ll h^{*}$. But the condition $L \gg h^{*}$ still must be saved.

At present, the theoretical and experimental methods of determination of averaged constants are elaborated for composite materials in the framework of this model. Especially, the progress in development of theoretical methods should be emphasized.

Model 2 is obtained as the result of utilization of the homogenization principle. Let us remember that it consists in changing the piece-wise homogeneous continuum by some homogeneous continuous continuum with the corresponding averaged properties within the framework of the model of anisotropic medium.

Finally, it seems to be expedient to note that model 2 is more approximate when comparing with model 1 . Therefore, the exactness of results obtained by model 2 can be estimated using the results obtained by model 1 .

Let us stop on some perspectives of developing based on model 2 approach. First of all, discreteness of structure of nanoformations as mechanical systems is of common knowledge. Also, when physical-mechanical properties of nanoformations are being determined, the concept of continualization is as a rule applied, and the discrete structure is approximately changed by a homogeneous continuous system (continuum). At that, the quantities are determined, which are peculiar for the continuum solid mechanics (Young modulus, Poisson ratio, proportional limit, yield strength, ultimate strength, corresponding to fracture strains, and so forth). Of course, in most cases of analysis of nanomaterials, the use of principle of continualization seems to be expedient, too.

Taking into account the insufficient level of studying the properties of nanoformations and the existence of, at present, quite good base of mechanical characteristics of microcomposite materials, let us adduce first the necessary facts from micromechanics of materials for the following comparative discussion. Among many important achievements of micromechanics of composite materials, let us show only two.

The first achievement is related to understanding the role of mechanical models: one and the same composite can be described by diverse microstructural models: from the complex multicontinuum models to the discrete models in the form of lattices [50-53]. Every model has some advantages, when either concrete problem is studied. The plenty of models testifies the achieved depth of understanding in micromechanics of materials.

The second achievement consists in that there is the big set of different mechanical characteristics for components of composite materials (matrixes, granules, fibers, and layers).

Below, as an example, the list for such set for aramid fibers (kevlars) is shown [54]:
"(1) density; (2) diameter of single fiber; (3) equilibrium humidity; (4) ultimate strength under tension; (5) elongation under break; (6) initial elastic modulus; (7) maximal elastic modulus; (8) elastic modulus under bending; (9) calculation modulus under axial tension; (10) dynamical elastic modulus; (11) part of strength in the loop from the ultimate strength under tension; (12) fatigue properties (number of cycles up to failure); (13) creep under loading up to $90 \%$ of the ultimate strength; (14) constant of friction."

At present, the information on mechanical characteristics of nanoformations is still insufficient, and the listed above example with fourteen characteristics of certain fiber can be understood as the very distant goal in nanomechanics.

Thus, aspect 3 can be, like aspect 2, related to arguments about similarity of all four parts of structural mechanics of materials, because two basic models of the continuum mechanics of these parts are identical. 


\section{Aspect 4: Allowance for the Edge and Near-the-Surface Effects}

The problem of allowance for the edge and near-the-surface effects is important for all of the parts of mechanics of materials. As a rule, analysis of this problem permits to estimate the validity of continuum models.

Remember that, in structural mechanics of composites (in the broad sense) and in mechanics of composite materials (in the more narrow sense), the principles of continualization and homogenization are utilized. According to the first one, the discrete structure is changed (modeling) by the continuous structure. According to the second one, the piecewise homogeneous structure is changed (modeling) by the homogeneous structure.

It is necessary to take into account that principles of continualization and homogenization are referring to modeling the properties of material as the infinite continuum.

When different problems of structural mechanics of materials (problems of statics, dynamics, stability, and fracture) are being studied, analysis is necessary to be carried out as a rule for the material occupying the finite volume, which is also characterized by the boundary surface. On the boundary surface, for all of the basic mechanical processes, some boundary conditions are formulated for the material. In this regard, the question on applicability of principles of continualization and homogenization near the boundary surface and on this surface arises. The answer to this question can be formulated as follows:

"near the boundary surface, the principles of continualization and homogenization do not work."

The proof of this statement seems to be quite evident, because near the boundary surface (under loading of arbitrary type) the representative volume of material is inherent in this material basic property-property of homogeneity of fields of macrostresses and macrostrains. The macrostresses and macrostrains are understood here as the stresses and strains within the framework of continuous system (medium), to which the initial system is reduced after application of continualization and homogenization principles.

Note also that, in composite materials, when the material is being modeled by the piecewise homogeneous medium, the inhomogeneous fields of stresses and strains near the boundary surface in each component (each homogeneous medium) arise as a rule. The statement above is true for all of the four scales mechanics (macro-, meso-, micro-, and nano-).

Below, inapplicability of principles of continualization and homogenization near the boundary surface is illustrated by an example of layered materials within the framework of micromechanics of composite materials. More specifically, let us consider the layered composite material formed of two alternating layers of constant thickness, which are made of materials with distinguishing properties.

In Figures 2 and 3, the two-dimensional representative area is shown, which includes the part of boundary surface also, that is, the representative volume near the boundary surface. At that, the dotted line denotes the exarticulation of the part of material near the boundary surface, and the size of the representative area side is $10\left(h_{1}+h_{2}\right)$, where $h_{1}$ and $h_{2}$ are the thicknesses of the forming of material layers. Thus, the sizes of representative area are distinguished one order from the structural components size $\left(h_{1}+h_{2}\right)$, which usually, in different publications, is assumed to be sufficient, when the sizes of representative area are being discussed.

It seems obvious that, owing to presence of boundary surface with arbitrary sizes of the representative area in Figures 2 and 3, the strains and stresses in the representative area are inhomogeneous. At that, this inhomogeneity will decrease with distance from the boundary surface. This phenomenon of inhomogeneity of fields is known in solid mechanics for homogeneous materials. In this case, the values of strains and stresses become more homogeneous, when moving off from the boundary surface.

When applied to statical problems, this phenomenon corresponds to the Saint-Venant edge effect.

When applied to problems of wave dynamics, this phenomenon corresponds to onset of surface waves with amplitude damping with moving off from the boundary surface.

The notion of surface instability for homogeneous anisotropic body (which corresponds to model 3) is seemingly introduced for the first time in [55]. Results of investigations of phenomenon of surface instability for different homogeneous materials with using strong $3 \mathrm{D}$ theories are represented along with other results in many monographs (e.g., starting with $[56,57]$ and finishing with $[58,59])$. In the work in [60], the phenomenon occurring near the boundary surface of elastic anisotropic body was called the skin effect.

Thus, in problems of statics, dynamics, and stability of mechanics of homogeneous materials (including model 3), the phenomenon consisting in the fields of inhomogeneous strains and stresses arising near the boundary surface of material, which damp quickly moving off from the boundary surface (skin effect, edge effect, and near-the-surface effect), is known quite well.

It is obvious that similar type effects take place both for materials with discrete structure (model 1 ) and for piece-wise homogeneous materials (model 2). For these materials, owing to existence of edge or near-the-surface effects, the additional complication arises-near the boundary surface, the principles of continualization and homogenization are inapplicable, because, including the boundary surface representative volume of arbitrary size, the fields of strains and stresses are inhomogeneous.

Taking into account the considerations above, it seems expedient to form the following conclusions.

Conclusion 1. For materials with discrete structure (model 1) and for piece-wise homogeneous materials (model 2), the study of edge effects can be carried out within the framework of initial models only because the principles of continualization and homogenization are here inapplicable.

Conclusion 2. For the convenience of studying, the edge and near-the-surface effects can be divided on effects near 
the boundary surface, which is parallel to the direction of prevailing reinforcement (the shown type in Figure 2), and effects near the boundary surface of the end face type are perpendicular to the direction of prevailing reinforcement (the shown type in Figure 3).

Conclusion 3. Analysis of edge and near-the-surface effects can be carried out within the model of homogeneous anisotropic body (model 3) also, but the results will be spurious as compared with analysis within the framework of models 1 and 2.

At present, a row of results is obtained in studying the edge and near-the-surface effects of the shown type in Figures 2 and 3 they are obtained within the framework of the model of piece-wise homogeneous material (model 2). The results are obtained for the model that corresponds to Conclusion 1.

Note that the strong method of solving the problems on near-the-surface effects in the case when the boundary surface is parallel to interfaces is proposed in the monograph [61] when applied to the layered materials of periodic structure. Here, the edge and surface effects are studied within the framework of wave dynamics: the propagation of surface waves along the plane boundary.

When applied to edge effects in the case when the boundary surface is perpendicular to interfaces in layered or fibrous materials, the results of studying the static problems of materials are stated in [9-20]. Here, only the simplest problems are studied, and the results are obtained using the numerical methods.

For the near-the-surface effects, when the boundary surface is parallel to interfaces, the results on constructing the surface instability of layered materials are stated in [57]. Here, for cases of the full contact of layers and the sliding layers, the method proposed in [61] was used. For the case of presence of cracks at interface, the numerical methods were used. These results correspond to fracture mechanics under compression, when the initial stage of fracture is the stability loss in the near-the-surface layers.

For the near-the-surface effects in fibrous unilateral composites, when the boundary surface is parallel to fibers, the results on constructing the surface instability are stated in [57]. These results correspond to fracture mechanics of fibrous materials under compression, when the initial stage of fracture is the stability loss of fibers in the near-the-surface area.

Let us show finally the exceptional example of composite material, in which the type of loading and the structure of composite are such that the edge and near-the-surface effects shown in Figures 2 and 3 do not arise. Consider a layered composite material composed of orthotropic layers of constant thickness. Layers differ by thickness and physicalmechanical properties. Symmetry axes of materials of layers coincide with the coordinate axes. So, the layered composite with arbitrary number of layers in the package is analyzed. Such a package is shown in Figure 4. It is assumed that at interfaces the conditions of continuity of displacement and stress vectors are fulfilled. The shown analysis rests valid for other boundary conditions at interfaces.

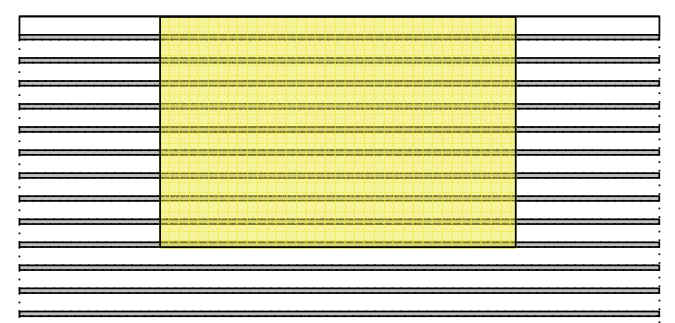

FIGURE 2: The layered material with boundary surface parallel to interfaces.

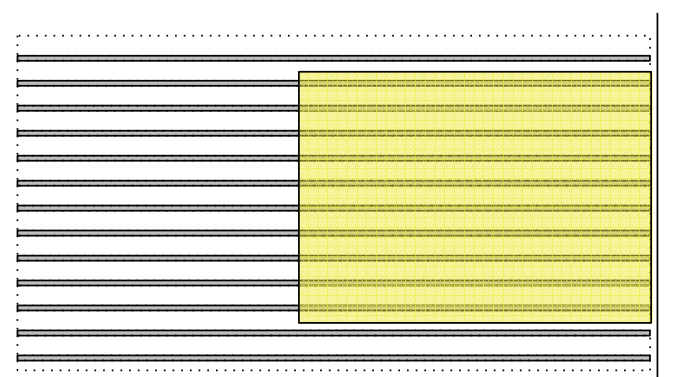

FIGURE 3: The layered material with boundary surface placed perpendicularly to interfaces.

The case of plane strain in the plane $x_{1} O x_{2}$ is considered, when compression along the axis $O x_{1}$ is realized by the rigid discs, which are shown in Figure 4 by rectangles of black color. It is assumed that the rigid discs provide the identical for all the layers shortening and the possibility of slipping motion of end faces of each layer along discs. Then, in each layer, the homogeneous stress-strain state is arising, different for different layers. In this way, the edge effects of the two above-considered types do not arise.

Note that at the end face of each layer the following boundary conditions $u_{1}=C x_{1} \quad(C=$ const $)$ and $\sigma_{12}=0$ are given. If the compression is realized by the uniformly distributed normal load, then within the framework of the plane strain state the edge effects near the end face will already arise.

The similar phenomenon of absence of edge effects arises also in the fibrous unilateral composite materials under their compression through the rigid discs along the reinforcing fibers. This phenomenon of homogeneous stress-strain state in the matrix and the fibers arises, when additionally assuming the coefficient of transverse expansion of the matrix and the fibers to be identical.

The shown above discussion of the edge and nearthe-surface effects forms a separate aspect of structural mechanics of materials, which provides the best understanding of results, which are obtained by means of principles of continualization and homogenization. This aspect testifies similarity of all four parts of structural mechanics of materials, including the nanomechanics of materials. 


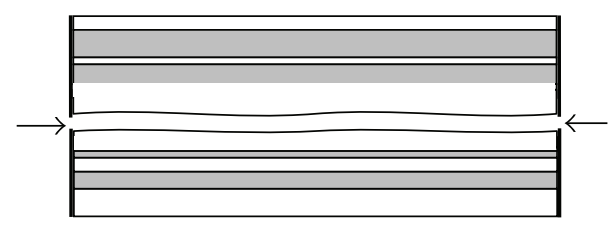

FIGURE 4: Layered composite material composed of orthotropic layers of constant thickness.
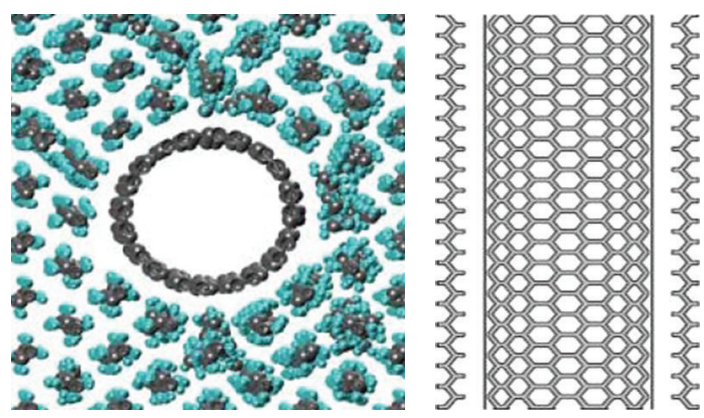

FIGURE 5: The molecular structure of nanocomposite crystallic polyethylene-CNT.

\section{Aspect 5: Phenomena at Interface in Nanocomposite Materials}

These phenomena arise in all of the kinds of composite materials and are studied very intensively. To analyze such phenomena, it seems convenient to introduce the notion of geometrical interface of nanoformation and polymeric matrix.

"The geometrical interface is meant to be the surface, sizes, and shape which are determined when nanoformations are being described in continuum approximation."

When the nanoformations and matrix are united into a nanocomposite, the phenomena occur at interfaces with participation of more deep-laid mechanisms that take place, for example, in the case of microcomposites.

The point is that in the general case the nanoformations consist of a system of curvilinear layers; in turn, each layer consists of a system of atoms, interaction among which is determined by force of interatomic interaction.

Therefore, when the nanoformations and matrix are being composed into a nanocomposite, seemingly, the interaction of atoms of the "end" layer of atoms on nanoformation with the neighboring atoms of polymeric matrix must occur owing to forces of interatomic interaction.

Thus, some intermediate layer arises from materials of nanoformations and polymeric matrix, inside which the interaction of atoms of nanoformations and polymeric matrix is observed. For example, in Figure 5, the molecular structure of nanocomposite crystallic polyethylene-CNT-is shown schematically.

Note that Figure 5 corresponds to [62] and is modified here to illustrate existence of intermediate layer discussed above. On the left side of Figure 5, the cross-section of nanocomposite is shown.

Let us note also that studying this phenomenon and finding the characterizing regularities seem to be the complex and urgent physical-chemical problem. The solution of this problem can be realized by representatives of corresponding scientific directions only.

To describe the phenomena in the intermediate layer within the framework of mechanics of nanocomposites in cases like those shown above, it is expedient to use the traditional approaches of mechanics developed early, when the related problems are being considered.

The tradition in mechanics in an analysis of phenomena occurring in the intermediate thin layers or on surfaces of thin bodies consists in

\section{"modeling these phenomena by certain boundary con-} ditions."

At that, the boundary conditions are transferred on certain in some sense close but the simpler surface.

Consider the following few examples.

Example 1. In the classical theory of wing flow, the boundary conditions on the wing surface are transferred on the wing chord.

Example 2. In the classical theory of contact interaction of elastic bodies, in the case when the stamp bottom has some deviations from the plane form, the boundary conditions are transferred on the plane boundary.

Example 3. In the problem of dynamical interaction of fluid and elastic (including thin-wall) bodies, the boundary conditions on vibrating interface are transferred at the fixed interface.

Taking into account these traditional, in mechanics approaches and practice of modeling of nanoformations and matrix by continuum systems, the following, corresponding to and adopted in mechanics, exactness approach can be proposed:

"it is expedient to model the phenomena occurring in the thin intermediate layer (nanoformation + polymeric matrix) by certain boundary conditions of interfacing of two media and translating these conditions at the geometrical interface "nanoformation matrix"'"

This approach has been proposed at the first time, seemingly, in [62].

Ascertainment of concrete structure of boundary conditions, reflecting the phenomena in intermediate layer, is still problematic, because physicists and chemists still have not built the sufficiently grounded theory of such phenomena.

Because establishing the concrete structure of boundary conditions at geometrical interface seems to be problematic, the development of bilateral estimates for these conditions becomes of special urgency. Such estimates permit to estimate also the values of corresponding quantities.

From the point of view of mechanics, the most stiff or perfect interfacing of two media (in this case, interfacing of 
the nanoformation and matrix) in the continuum description corresponds to the conditions of total contact (continuity of stress and displacement vectors) at the interface; the most compliant or imperfect interfacing of two media corresponds to the conditions of sliding contact.

Thus, the study of nanocomposites under two mentioned types of boundary conditions at geometrical interface enables to obtain the bilateral estimates for quantities of interest, when various physical-chemical mechanisms are being manifested at the interface.

It should be also stressed that numerous problems exist in mechanics of microcomposites that are associated with necessity to provide the corresponding adhesion strength at interface. These problems are arising because of the existence of various mechanisms at interface. But these mechanisms in microcomposites are not linked with the interatomic forces action, contrary to nanocomposites, in which this action can be essential.

\section{Aspect 6: The Validity Ranges of Continuum Mechanics of Materials}

Such an aspect exists in every physical theory. It forms the necessary element in the theory and, of course, should be discussed when applied to the structural mechanics of materials. Below, the reasons and information are expounded, arising in analysis of the validity ranges of continuum mechanics of materials.

Let us note that undoubtedly the strong and full solution of the problem of validity ranges is difficult to realize from mathematical point of view. In this regard, below, it is only one among many variants of its approximate solution and qualitative discussion that is considered.

Remember that, in mechanics of composite materials, the basic relationships of continuum mechanics of materials are used to describe the deformation of matrix and each reinforced element. Note also that validity of continuum mechanics of materials in cases of macro-, meso-, and micromechanics is analyzed quite well. Here, the case of nanomechanics is discussed.

Return now to relationships (1) and (3) and Figure 1; from which it follows that the structural nanomechanics studies the materials, in which the reinforcing particles can reach sizes not less than $1 \mathrm{~nm}$. At that, the distance between the atom planes has as a rule the order of one or more angstroms. It is accepted that atoms have also diameters of the order of angstrom.

The typical example of the filler used to produce the nanocomposites is the carbon nanotube-single-walled (SWCNT) or multiwalled (MWCNT) [63]. It is well known that the SWCNT is the carbon molecular layer, consisting of ordered (zig-zag, armchair, and chiral) structure of carbon atoms, which is as if rolled into the cylindrical tube. From the point of view of molecular physics, the carbon nanotube is the discrete system consisting of particles (atoms) the sizes of which have the order of 1.5 angstrom; at that, the distance between particles reaches the order of $1 \mathrm{~nm}$. Interaction among elements of this discrete system (among carbon atoms) is put into effect by nonmechanical way, because the material medium among separate carbon atoms is absent. Interaction among neighboring carbon atoms in the one atom layer (SWCNT) as well as the interaction among carbon atoms in neighboring layers (MWCNT) is put into effect by the forces of interatomic interaction, which is described by different potentials.

At present, in overwhelming number of theoretical publications, to determine the properties of carbon nanotubes, the approaches of molecular structural physics are used. They are based on the Cauchy-Born method [7] with using a different potential, describing the interatomic interaction (e.g., potentials in the Brenner form [64], Tersoff-Brenner form [65, 66], modified Morse potential [67], and so on).

As a result of such approaches, the peculiars for continuum solid mechanics quantities are obtained: the values of Young modulus, Poisson ratio, and others. In this way, the continualization of discrete system is realized, when the interaction among single elements (single atoms) occurs owing to forces of interatomic interaction.

Note that attempt to describe the deformation of reinforcing elements, especially nanotubes, within the framework of continuum solid mechanics, seems to be perspective and probably solely expedient, because it must correlate with description of deformation of matrix, for which the continuum approach is peculiar.

Taking into account the abovementioned, the problem of validity of basic relationships of continuum solid mechanics in the study of mechanical processes on the nanolevel (3) can be formulated as follows.

Which mechanical fields can be studied on the nanolevel (3), assuming the system in hand as the continuum or applying the basic relationships of continuum solid mechanics?

At that, it should be noted that the mechanical fields (stress and strain fields) in a nanoformation (e.g., in the nanotube) change by spatial variables.

Introduce, therefore, the geometrical parameter $L$, which characterizes the mechanical processes (characterizes the variability of mechanical fields by spatial variables) and has the dimension of length. In the case of statical problems, the parameter $L$ corresponds to the minimal distances, on which the stress and strain fields change significantly; in the problems of wave propagation, the parameter $L$ corresponds to the wavelength, and in the stability problems it corresponds to the wavelength of stability loss mode.

Thus, the problem of validity ranges is equivalent to the problem of determination of ranges for parameter $L$, which provide possibility to study the mechanical fields on the nanolevel (3) using the basic relationships on continuum solid mechanics (i.e., assuming the discrete system in hand as the continuum and homogeneous one).

To solve this problem, it seems necessary to use the introduced before and the characterized internal structure of material geometrical parameter $h$. Also, the geometrical parameter $h^{*}$ seems expedient to be introduced. It characterizes the mean value of distances between centers of particles in the internal structure of material. 
Taking into account the information above on atom sizes and distances between atoms, it can be assumed that parameters $h$ and $h^{*}$ have, when applied to nanoformations the following values:

$$
h \approx 1.5 \text { angstrom, } \quad h^{*} \approx 1 \mathrm{~nm} .
$$

The values in (12) can be chosen in a different way. For example, the value $h \approx 1.5$ angstrom corresponds to the carbon atom diameter.

Qualitative and partially quantitative solutions of the problem under consideration can be carried out by introducing the model of anisotropic homogeneous body. It is necessary for applicability of this model that the parameter $L$ (characterizing the variability of mechanical fields by the spatial variables) by more essentially quantity as compared with parameters $h$ and $h^{*}$ (characterizing the structure of composite material). This situation is provided by fulfilling the following conditions:

$$
L \gg h, \quad L \gg h^{*} .
$$

So, it follows from (12) and (13) that mechanical fields on nanolevel can be studied with utilization of the basic relationships of continuum solid mechanics, if the geometric parameter $L$ meets the condition

$$
L \gg 1 \mathrm{~nm} .
$$

If the condition "》" (more essentially) is fulfilled when the quantities are distinguished even on one order, then condition (14) can be represented in the form

$$
L \geq 10 \mathrm{~nm} .
$$

Thus, when the mechanical fields, for which the parameter $L$ fulfills either condition (14) or (15), are being studied, the model of continuum can be used (or the basic relationships of continuum solid mechanics can be used, which in this case is identical) for nanocomposite materials taking into account their real atom structure.

The condition (14) or (15) determines the validity ranges of continuum solid mechanics in studying the mechanical fields in composite material components (i.e., separately in the matrix material and separately in the material of each reinforcing element for materials with other structural levels (macro-, meso-, and micro-) within the framework of structural mechanics of materials).

The condition (14) or (15) determines also the sufficiently wide frame of mechanical fields, including fields under action of quite high frequency in dynamical problems and under quite short-wave modes of stability loss.

Finally, note once more that the abovestated analysis is approximate and seemingly has substantially the qualitative character. Nevertheless, it is sufficiently expedient, because the problem of validity ranges of continuum mechanics in analysis of mechanical fields in the modern structurally inhomogeneous materials has a character of traditionally constantly discussed problem.

So, the above-considered aspect of mechanics of nanocomposite materials allows to elaborate the constructive tool for separation of objects of nanomechanics from the general set of objects, which includes the micro-, meso-, and macroobjects too. This aspect can, like aspect 1 , be related to those ones which show the distinction between mechanics of nanocomposite materials and mechanics of composite materials of the higher structural levels.

\section{Aspect 7: Approaches "Bottom-Up" and "Top-Down"}

The view on mechanics of nanocomposite materials in the light of approaches "bottom-up" and "top-down" seems very meaningful, since it highlights the specificity of nanolevel composite materials.

In technology, the two approaches "bottom-up" and "top-down" are generally known. Sometimes, "bottom-up" is commented as "nucleation and growth," and "top-down" is commented as "comminution and dispersion" [68].

The approach "bottom-up" consists in the making of materials, starting with the smallest particles up to more massive formations. In this approach, the most essential is the basis: the aggregate of smallest particles and their character. The basis forms the foundation for constructing the more massive volumes of material. This basis is called the bottom.

The approach "top-down" consists in the making of materials, starting with the large volumes of material (bulk materials, source of raw materials) in direction towords the smaller formations (pieces) of material. The rough material is pressed, cut, found, or in some different way formed into pieces or products. In this approach, the most important are the tool resources, by which the lower limit in sizes of product or material piece is determined.

But mechanics as one of the oldest sciences has also the "canonical" terminology; thus, the situation needs some discussion. In mechanics as science, the basic approach can be apparently meant as constructing the models of phenomena, processes, and materials. With allowance for the historical experience, the models in mechanics of materials are developed in direction of studying the structure of material with more and more fine scale of internal structure.

When the terminology above is being considered, it seems expedient to refer to the review in [69], which is published in 2005 and entitled "Nanocomposites in context (review)." This is consonant with the well-known review "Composites in context" [70], published in 1985. Let us cite for illustration one sentence from [69]:

Scientists and engineers working with fiber-reinforced composites have practiced this bottom-up approach in processing and manufacturing for decades. When designing a composite the material properties are tailored for the desired performance across various length scales. From selection and processing of matrix and fiber materials, and design and optimization of the fiber/matrix interface/interphase at the sub-micron scale to the manipulation of yarn bundles in 2-D and 3-D textiles to the lay-up of laminae in laminated composites and finally the net-shape forming of the macroscopic composite part, 


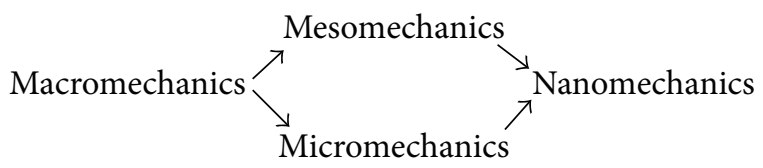

FIGURE 6

the integrated approach used in composites processing is a remarkable example in the successful use of the "bottom-up" approach.

This sentence testifies that the term "bottom-up" is used in technology and it was used in the making of the microcomposites (they correspond to microlevel and are studied in micromechanics).

Let us separate three moments.

Moment 1 . When the nanocomposites are being made (taking into account the used, in technology, terminology), the following chain (sequence) in formation of material is realized "aggregate of atoms (bottom) $\rightarrow$ nanomolecules, nanotubes $\rightarrow$ nanoformations (nanoparticles, nanoplates; nanowires, nanobundles, nanoropes $\rightarrow$ nanofibers $) \rightarrow$ nanocomposite."

Thus, in technology of making the nanocomposites, the "bottom-up" approach is realized by making the material starting with the aggregate of atoms. This terminology is found everywhere in publications on nanotechnology and nanomaterials. So, in the book [70], in the approaches "bottom-up" and "top-down" subsection 1.9 is devoted. Note also that the term "bottom" was used in the Feynman famous lecture in [71].

Moment 2. In structural mechanics of materials, the basic approach consists in constructing the chain of models in direction of taking into account the more and more fine internal structure of materials. This sequential chain can be sketched out in Figure 6.

Thus, it can be thought that the terms bottom-up approaches and descending approaches have some interpretation in the structural mechanics of materials. So, the term bottom-up approaches corresponds to motion from the left to the right end of the level scale from the chain above. The term descending approaches corresponds to motion from the right to the left end of the level scale from the chain above. Let us remember that the right end corresponds to approaches, where taking into account the atom structure is obligatory.

Moment 3. Despite some distinction of terminology in technology and structural mechanics of materials, investigations on mechanics of nanocomposites are carried out within the framework of nanolevel. Therefore, at the first stage, they are carried out taking into account the atom structure under different ways of its description.

In correspondence $[1,2,5,62]$, it is proposed in mechanics of nanocomposites to apply the approaches and methods of structural macro-, meso-, and micromechanics of materials, in which the matrix and fillers are described within the framework of continuum representation. In this regard, the approaches and methods of micromechanics of materials are meant to be preferable because they are the closest in the hierarchy of structural components.

Thus, the aspect in hand is useful for mechanics of nanocomposite materials, because it allows to segregate this part of structural mechanics of material among other parts.

\section{Aspect 8: Basic Approaches in Mechanics of Nanocomposite Materials}

The challenging nanocomposites can be defined as the produced, at present and in the future, materials that can be applied in structural elements with allowance for features of loading and optimal correspondence of functioning of the elements under this loading.

Such optimality is realized by means of creating the anisotropy of deformation and strength properties of structural elements. The possibility of this creation is one of the most characteristic features of composites along with the high strength-to-weight ratio and high modularity.

These special properties can be formed in nanocomposites only by the straightening in directions of prevailing reinforcing the quite elongated and straightline nanoformations (CNT, nanoropes, nanofibers, and so on) as fillers, which should be coordinated with the force fluxes and be highmodulus ones.

Like the cases of macro- and microcomposites, forecasting the behavior of nanocomposites under mechanical actions can be realized mainly thanks to the theoretical studies. This is why significance of such studies is difficult to overestimate. Especially, for producing, in the future, nanocomposites, we have to take into account the strategy of technology.

It is worthy to note that at present the overwhelming number of publications on nanocomposites is devoted to analysis of nanocomposites under dispersive distribution in matrix of the comparatively short nanoformations as fillers. At that, the clearly expressed directions of reinforcing are absent. These dispersive nanocomposites can be classified as the type of hardened matrix, which is frequently reflected in terminology of publications.

But this type of engineering cannot define the progress of perspective nanocomposites, in which reinforcing is coordinated with loading.

The considerations above testify that in constructing the foundations of mechanics of nanocomposites with polymeric matrix the quite substantiated is the basic approach, which can be meant as "set of concepts, models and statements of problems, development of methods of analysis, and obtaining the new results, which are adequate to phenomena of interest in perspective nanocomposite materials under mechanical actions."

The formulated basic approach consists of the following four parts.

(i) Part I. Modeling of the nanoformations by the linear elastic isotropic homogeneous body with averaged values of elastic constants, which are obtained with attracting the concept of continualization from results 
taking into account the action of forces of interatomic interaction.

(ii) Part II. Modeling of the polymeric matrix (binder) by the linear isotropic homogeneous elastic or viscoelasticbody. The similar modeling was used traditionally in constructing the foundations of mechanics of microcomposites. Under moderate temperatures or under comparatively short-time loading, a polymeric matrix in nanocomposites can be modeled by the linear isotropic elastic body.

(iii) Part III. Modeling of the interaction of nanoformations and polymeric matrix (in the thin intermediate layer with allowance for forces of interatomic interaction) by certain boundary conditions with transferring these conditions on the geometric interface, using the boundary conditions of perfect contact (continuity of stress and displacement vectors) and boundary conditions of sliding contact for bilateral estimates of quantities under consideration.

(iv) Part IV. Determination of the averaged values of elastic constants for nanocomposites using various methods of homogenization, which provides the transition to mechanics of structural members from nanocomposites.

In this way, within the framework of the basic approach, the different problems of statics, dynamics, stability, and fracture of nanocomposite materials and structural elements made of these materials are carried out.

It was mentioned before, in analysis of problems of mechanics of nanocomposites, that the model of piece-wise homogeneous medium (after realization of the concept of continualization for nanofillers) and the model of homogeneous anisotropic body with averaged elastic constants (after realization of the concept of continualization for nanofillers and the following realization of the concept of homogenization for the obtained piece-wise homogeneous medium) are used.

In analysis of problems in mechanics of structural elements made of nanocomposites, the application of the model of anisotropic homogeneous body with averaged values of elastic constants seems to be preferable.

Of course, in this part of mechanics, an analysis of multilayered constructions (e.g., constructing the models and theories of multilayered rods, plates, and shells) is actual. In this case, for each single element of construction (for each layer), the model of anisotropic homogeneous body with averaged elastic constants is used.

Within the framework of the basic approach, a row of new results is obtained.

It is necessary to point out that actually a number of reviews on different problems of mechanics of nanocomposites are published $[1,2,5,62,69,72-77]$.

Note also that the essential moment in the basic approach is the second position of part III, in concordance with which the bilateral estimates for quantities of interest are expedient to have. They are based on study of boundary conditions of perfect contact and sliding contact on the geometrical interface "filler-binder." The obtained, in such a way, bilateral estimates enable to determine values of quantities of interest for almost all various physical-chemical processes occurring in the intermediate layer between the filler and the polymeric matrix.

\section{Aspect 9: Transition from Mechanics of Nanomaterials to Mechanics of Nanostructural Members}

The abovestated considerations on mechanics of nanocomposites form the theoretical prerequisites for studying the basic problems arising in this part of mechanics: statical and dynamical problems, problems of stability, and fracture mechanics problems. In such studies, the statements of problems and methods of solving analogous to the approaches that are developed in mechanics of microcomposite can be used (see, e.g., multivolume editions [9-16, 23-49, 77]). In these studies, the model of piece-wise homogeneous medium and the model of homogeneous medium with averaged properties can be traditionally used.

In the studies within the framework of mechanics of structural members, it seems mostly promising and may be solely possible to apply the approach when the nanocomposite (piece-wise material) is changed on the homogeneous material with averaged properties.

Thus, in problems of mechanics of structural members made of nanocomposites, first the principle of homogenization is expedient to use and then to consider the nanocomposite as the homogeneous material with averaged properties.

When applied to determination of averaged properties of nanocomposites, two approaches can be seemingly singled out as follows:

Approach 1: determination of averaged constants within the framework of the model of anisotropic homogeneous body (the structural model of the first order),

Approach 2: determination of averaged parameters within the framework of the structural models of the second order.

As applied to nanocomposites, when the averaged constants are being determined within the framework of the model of anisotropic elastic homogeneous body, the statements of problems and methods of studying that are developed for granular, fibrous, and layered microcomposites of determined and stochastic structure can be applied, as they. These statements and methods are expounded in numerous publications (e.g., in [9-20]).

It is necessary to note that in this case the values of averaged constants are asymptotically exact and follow from the rigorous results obtained within the framework of threedimensional theory under some conditions.

When applied to theory of wave propagation, these conditions correspond to the situation, when the ratios of geometrical parameter characterizing the internal structure of nanocomposite to the wavelength are tending to zero, that is, as if corresponding to the long-wave approximation. 
Similar conditions are applied in other problems of mechanics of nanocomposite materials.

Thus, this aspect can be related to arguments about similarity of all four parts of structural mechanics of materials, because at present all known ways of transition from mechanics of materials to mechanics of structural members in these parts are identical.

\section{Final Remarks}

Thus, the considered aspects of mechanics of nanocomposite materials and structural members permit to outline the similarities and distinctions of this part of structural mechanics when comparing with the other three parts-macro-, meso-, and micromechanics of materials and structural members.

The irony of fate for mechanics of nanocomposite materials and structural members consists in that similarities are essentially more studied, and they determine the tight link among all of the four parts, which is little known beyond the mechanics of materials and structural members.

Sometimes, the nature of human perception is that distinctions are fixed more often and with significantly less impediments.

Therefore, the nanomechanics in whole is represented up to this time through the prism of distinctions despite the presence of a big corpus of similarities.

\section{References}

[1] A. N. Guz, J. J. Rushchitsky, and I. A. Guz, Introduction to Mechanics of Nanocomposites, Akademperiodika, Kiev, Ukraine, 2010 (Russian).

[2] A. N. Guz, A. A. Rodger, and I. A. Guz, "Developing a compressive failure theory for nanocomposites," International Applied Mechanics, vol. 41, no. 3, pp. 233-255, 2005.

[3] I. A. Guz, J. J. Rushchitsky, and A. N. Guz, "Mechanical models in nanomaterials," in Handbook of Nanophysics. Principles and Methods, K. D. Sattler, Ed., vol. 1, chapter 24, pp. 24.1-24.18, Taylor and Francis Publisher (CRC Press), Boca Raton, Fla, USA, 2010.

[4] A. N. Guz and Y. Y. Rushchitskii, "Nanomaterials: on the mechanics of nanomaterials," International Applied Mechanics, vol. 39, no. 11, pp. 1271-1293, 2003.

[5] I. A. Guz, A. A. Rodger, A. N. Guz, and J. J. Rushchitsky, "Developing the mechanical models for nanomaterials," Composites A, vol. 38, no. 4, pp. 1234-1250, 2007.

[6] C. Truesdell, A First Course in Rational Continuum Mechanics, The John Hopkins University, Baltimore, Md, USA, 1972.

[7] M. Born and K. Huang, Dynamical Theory of Crystal Lattices, Oxford University Press, Oxford, UK, 2007.

[8] L. I. Slepyan, Mechanics of Cracks, Sudostroenie, Leningrad, Russia, 1990 (Russian).

[9] V. T. Golovchan, Ed., Statics ofMaterials, vol. 1 of Mechanics of Composites, Naukova Dumka, Kiev, Ukraine, 1993 (Russian).

[10] N. A. Shul'ga, Ed., Dynamics and Stability of Materials, vol. 2 of Mechanics of Composites, Naukova Dumka, Kiev, Ukraine, 1993 (Russian).

[11] L. P. Khoroshun, Ed., Statistical Mechanics and Effective Properties of Materials, vol. 3 of Mechanics of Composites, Naukova Dumka, Kiev, Ukraine, 1993 (Russian).
[12] A. N. Guz and S. D. Akbarov, Eds., Mechanics of Materials with Curved Structures, vol. 4 of Mechanics of Composites, Naukova Dumka, Kiev, Ukraine, 1995 (Russian).

[13] A. A. Kaminskii, Ed., Fracture Mechanics, vol. 5 of Mechanics of Composites, Naukova Dumka, Kiev, Ukraine, 1996 (Russian).

[14] N. A. Shul'ga and V. T. Tomashevsk, Eds., Process-Induced Stresses and Strains in Materials, vol. 6 of Mechanics of Composites, Naukova Dumka, Kiev, Ukraine, 1997 (Russian).

[15] A. N. Guz, A. S. Kosmodamianskii, and V. P. Shevchenko, Eds., Stress Concentration, vol. 7 of Mechanics of Composites, Naukova Dumka, Kiev, Ukraine, 1998 (Russian).

[16] Ya. M. Grigorenko, Ed., Statics of Structural Members, vol. 8 of Mechanics of Composites, Naukova Dumka, Kiev, Ukraine, 1999 (Russian).

[17] V. D. Kubenko, Ed., Dynamics of Structural Members, vol. 9 of Mechanics of Composites, Naukova Dumka, Kiev, Ukraine, 1999 (Russian).

[18] I. Yu. Babich, Ed., Stability of Structural Members, vol. 10 of Mechanics of Composites, Naukova Dumka, Kiev, Ukraine, 2001 (Russian).

[19] Ya. M. Grigorenko and Yu. N. Shevchenko, Eds., Numerical Methods, vol. 11 of Mechanics of Composites, Naukova Dumka, Kiev, Ukraine, 2002 (Russian).

[20] A. N. Guz and L. P. Khoroshun, Eds., Applied Investigations, vol. 12 of Mechanics of Composites, Naukova Dumka, Kiev, Ukraine, 2003 (Russian).

[21] A. N. Guz, "Three-dimensional theory of stability of a carbon nanotube in a matrix," International Applied Mechanics, vol. 42, no. 1, pp. 19-31, 2006.

[22] I. A. Guz, A. A. Rodger, A. N. Guz, and J. J. Rushchitsky, "Predicting the properties of micro- and nanocomposites: from the microwhiskers to the bristled nano-centipedes," Philosophical Transactions of the Royal Society A, vol. 366, no. 1871, pp. 18271833, 2008.

[23] A. G. Metcalfe, Ed., Interfaces in Metal Matrix Composites, vol. 1 of Composite Materials, Academic Press, New York, NY, USA, 1974.

[24] G. P. Sendeckyi, Ed., Mechanics of Composite Matreials, vol. 2 of Composite Materials, Academic Press, New York, NY, USA, 1974.

[25] B. Noton, Ed., Application of Composite Materials in Technics, vol. 3 of Composite Materials, Academic Press, New York, NY, USA, 1974.

[26] B. Krider, Ed., Composite Materials with Metallic Matrix, vol. 4 of Composite Materials, Academic Press, New York, NY, USA, 1974.

[27] L. J. Broutman, Ed., Fracture and Fatigue, vol. 5 of Composite Materials, Academic Press, New York, NY, USA, 1974.

[28] E. P. Plueddemann, Ed., Interfaces in Polymer Matrix Composites, vol. 6 of Composite Materials, Academic Press, New York, NY, USA, 1974.

[29] C. C. Chamis, Ed., Structural Design and Analysis-Part 1, vol. 7 of Composite Materials, Academic Press, New York, NY, USA, 1975.

[30] C. C. Chamis, Ed., Structural Design and Analysis-Part 2, vol. 7 of Composite Materials, Academic Press, New York, NY, USA, 1975.

[31] T.-W. Chou, Ed., Fiber Reinforcements and General Theory of Composites, vol. 1 of Comprehensive Composite Material, Elsevier, London, UK, 2006. 
[32] R. Talreja and J. A. Mänson, Eds., Polymer Matrix Composites, vol. 2 of Comprehensive Composite Material, Elsevier, London, UK, 2006.

[33] T. W. Clyne, Ed., Metal Matrix Composites, vol. 3 of Comprehensive Composite Material, Elsevier, London, UK, 2006.

[34] R. Warren, Ed., Carbon/Carbon, Cement and Ceramic Composites, vol. 4 of Comprehensive Composite Material, Elsevier, London, UK, 2006.

[35] L. Carlsson, R. L. Crane, and K. Uchino, Eds., Test Methods, Nondestructive Evaluation, and Smart Materials, vol. 5 of Comprehensive Composite Material, Elsevier, London, UK, 2006.

[36] W. G. Bader, K. Kedsvard, and Y. Sawada, Eds., Design and Applications, vol. 6 of Comprehensive Composite Material, Elsevier, London, UK, 2006.

[37] I. Milne, R. O. Ritchie, and B. Karihaloo, Eds., Structural Integrity Assessment-Examples and Case Studies, vol. 1 of Comprehensive Structural Integrity, Elsevier, London, UK, 2006.

[38] B. Karihaloo and W. G. Knauss, Eds., Fundamental Theories and Mechanisms of Failure, vol. 2 of Comprehensive Structural Integrity, Elsevier, London, UK, 2006.

[39] R. de Borst and H. A. Mang, Eds., Numerical and Computational Methods, vol. 3 of Comprehensive Structural Integrity, Elsevier, London, UK, 2006.

[40] R. O. Ritchie and Y. Murakami, Eds., Cyclic Loading and Fatigue, vol. 4 of Comprehensive Structural Integrity, Elsevier, London, UK, 2006.

[41] A. Saxena, Ed., Creep and High-Temperature Failure, vol. 5 of Comprehensive Structural Integrity, Elsevier, London, UK, 2006.

[42] J. Petit and P. Scott, Eds., Environmentally Assisted Fracture, vol. 6 of Comprehensive Structural Integrity, Elsevier, London, UK, 2006.

[43] R. A. Ainsworth and K.-H. Schwalbe, Eds., Practical Failure Assessment Methods, vol. 7 of Comprehensive Structural Integrity, Elsevier, London, UK, 2006.

[44] W. Gerberich and W. Yang, Eds., Interfacial and Nanoscale Failure, vol. 8 of Comprehensive Structural Integrity, Elsevier, London, UK, 2006.

[45] Y.-W. Mai and S.-H. Teoh, Eds., Bioengineering, vol. 9 of Comprehensive Structural Integrity, Elsevier, London, UK, 2006.

[46] Indexes, vol. 10 of Comprehensive Structural Integrity, Elsevier, London, UK, 2006.

[47] L. P. Khoroshun, Ed., Mechanics of Materials, vol. 1 of Mechanics of Composite Materials and Structural Members, Naukova Dumka, Kiev, Ukraine, 1982 (Russian).

[48] Ya. M. Grigorenko, Ed., Mechanics of Structural Members, vol. 2 of Mechanics of Composite Materials and Structural Members, Naukova Dumka, Kiev, Ukraine, 1982 (Russian).

[49] Applied Investigations, vol. 3 of Mechanics of Composite Materials and Structural Members, Naukova Dumka, Kiev, Ukraine, 1983 (Russian).

[50] C. Cattani and J. J. Rushchitsky, Wavelet and Wave Analysis as Applied to Materials with Micro or Nanostructures, World Scientific Publishing, Singapore, 2007.

[51] Y. Y. Rushchitskii, "Extension of the microstructural theory of two-phase mixtures to composite materials," International Applied Mechanics, vol. 36, no. 5, pp. 586-614, 2000.

[52] J. J. Rushchitsky and S. I. Tsurpal, Waves in Materials with Microstructure, SP Timoshenko Institute of Mechanics, Kiev, Ukraine, 1998 (Russian).
[53] A. Bedford, G. S. Drumheller, and H. J. Sutherland, "On modeling the dynamics of composite materials," in Mechanics Today, S. Nemat-Nasser, Ed., vol. 3, pp. 1-54, Pergamon Press, New York, NY, USA, 1976.

[54] H. S. Katz and J. V. Milewski, Eds., Handbook of Fillers and Reinforcements for Plastics, Van Nostrand Reinhold Company, New York, NY, USA, 1978.

[55] M. A. Biot, "Surface instability in finite anisotropic elasticity under initial stress," Proceedings of the Royal Society A, vol. 273, no. 1354, pp. 329-339, 1963.

[56] A. N. Guz, Fundamentals of Three-Dimensional Theory of Stability of Deforming Bodies, Vyshcha Shkola, Kiev, Ukraine, 1986 (Russian).

[57] A. N. Guz, Fundamentals of Three-Dimensional Theory of Stability of Deformable Bodies, Springer, Berlin, Germany, 1999.

[58] A. N. Guz, Ed., Fracture in Structure of Material, vol. 1 of Fundamentals of Fracture Mechanics of Composites under Compression, Litera, Kiev, Ukraine, 2008 (Russian).

[59] A. N. Guz, Ed., Related Mechanisms of Fracture, vol. 2 of Fundamentals of Fracture Mechanics of Composites under Compression, Litera, Kiev, Ukraine, 2008 (Russian).

[60] M. A. Biot, "Fundamental skin effect in anisotropic solid mechanics," International Journal of Solids and Structures, vol. 2, no. 4, pp. 645-663, 1966.

[61] N. A. Shulga, Foundations of Mechanics of Layered Media of Periodic Structure, Naukova Dumka, Kiev, Ukraine, 1981 (Russian).

[62] A. N. Guz, J. J. Rushchitsky, and I. A. Guz, "Establishing fundamentals of the mechanics of nanocomposites," International Applied Mechanics, vol. 43, no. 3, pp. 247-271, 2007.

[63] S. C. Tjong, Carbon Nanotube Reinforced Composites: Metal and Ceramic Matrices, Wiley-VCH, Weinheim, Germany, 2009.

[64] D. W. Brenner, "Empirical potential for hydrocarbons for use in simulating the chemical vapor deposition of diamond films," Physical Review B, vol. 42, no. 15, pp. 9458-9471, 1990.

[65] J. Tersoff, "Empirical interatomic potential for carbon, with applications to amorphous carbon," Physical Review Letters, vol. 61, no. 25, pp. 2879-2882, 1988.

[66] J. Tersoff, "New empirical approach for the structure and energy of covalent systems," Physical Review B, vol. 37, no. 12, pp. 69917000, 1988.

[67] E. Hernández, C. Goze, P. Bernier, and A. Rubio, "Elastic properties of single-wall nanotubes," Applied Physics A, vol. 68, no. 3, pp. 287-292, 1999.

[68] J. Ramsden, Nanotechnology, Ventus Publishing ApS, Copenhagen, Denmark, 2010.

[69] E. T. Thostenson, C. Li, and T.-W. Chou, "Nanocomposites in context," Composites Science and Technology, vol. 65, no. 3-4, pp. 491-516, 2005.

[70] A. Kelly, "Composites in context," Composites Science and Technology, vol. 23, no. 3, pp. 171-199, 1985.

[71] N. Wilson, K. Kannangara, G. Smith, M. Simmons, and B. Raguse, Nanotechnology: Basic Science and Emerging Technologies, Chapman and Hall/CRC, Boca Raton, Fla, USA, 2002.

[72] V. A. Buryachenko, A. Roy, K. Lafdi, K. L. Anderson, and S. Chellapilla, "Multi-scale mechanics of nanocomposites including interface: experimental and numerical investigation," Composites Science and Technology, vol. 65, no. 15-16, pp. 2435-2465, 2005. 
[73] A. A. Guz, J. J. Rushchitsky, and I. A. Guz, "Comparative computer modeling of carbon-polymer composites with carbon or graphite microfibers or carbon nanotubes," Computer Modeling in Engineering and Sciences, vol. 26, no. 3, pp. 139-156, 2008.

[74] A. K.-T. Lau and D. Hui, "The revolutionary creation of new advanced materials_carbon nanotube composites," Composites $B$, vol. 33, no. 4, pp. 263-277, 2002.

[75] H. S. Nalwa, Handbook of Nanostructured Materials and Nanotechnology, vol. 3, Academic Press, San Diego, Calif, USA, 2000.

[76] D. Srivastava, C. Wei, and K. Cho, "Nanomechanics of carbon nanotubes and composites," Applied Mechanics Reviews, vol. 56, no. 2, pp. 215-229, 2003.

[77] Y.-W. Mai and Z. Yu, Eds., Polymer Nanocomposites, Woodhead Publishing Limited, Cambridge, UK, 2009. 

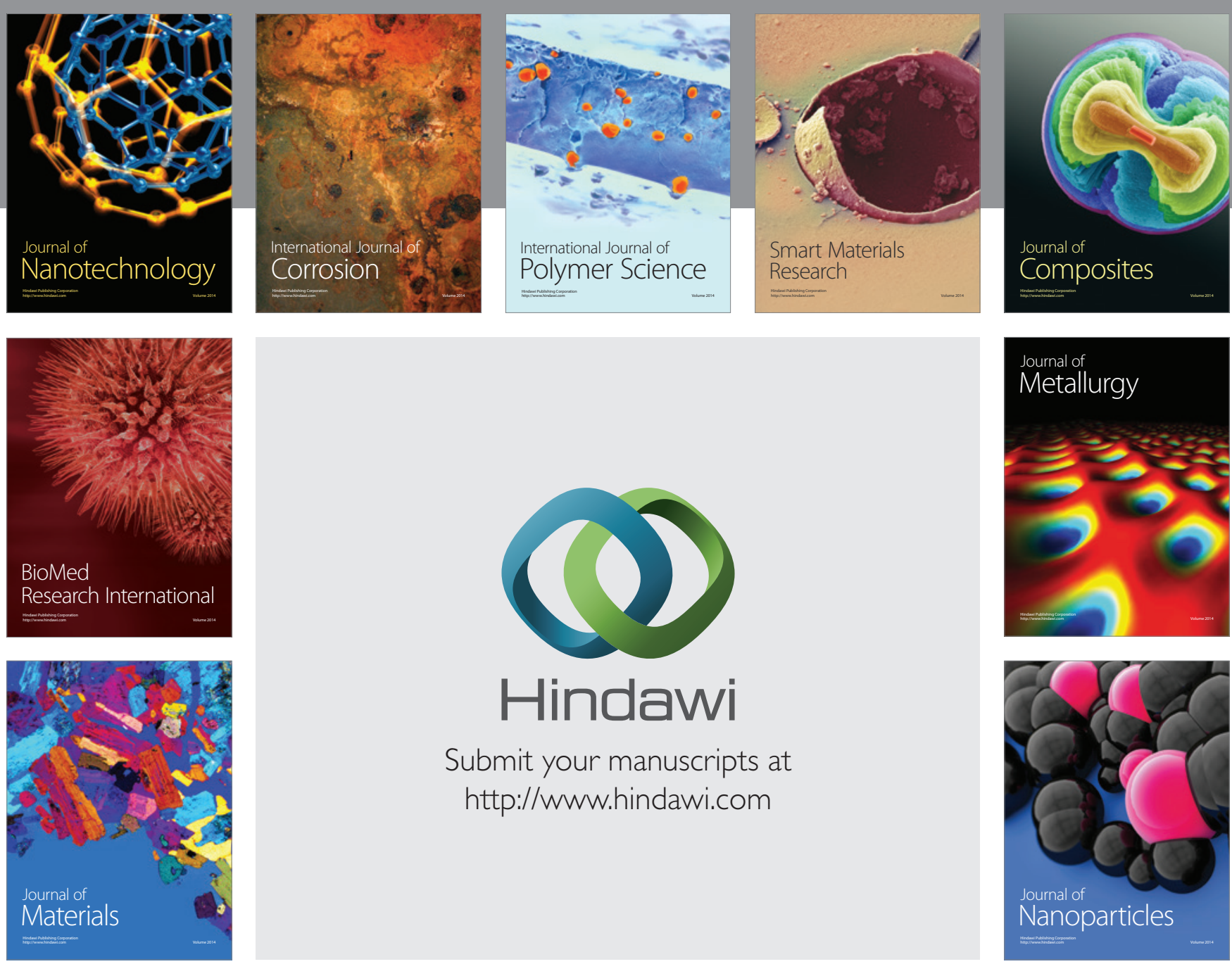

Submit your manuscripts at http://www.hindawi.com
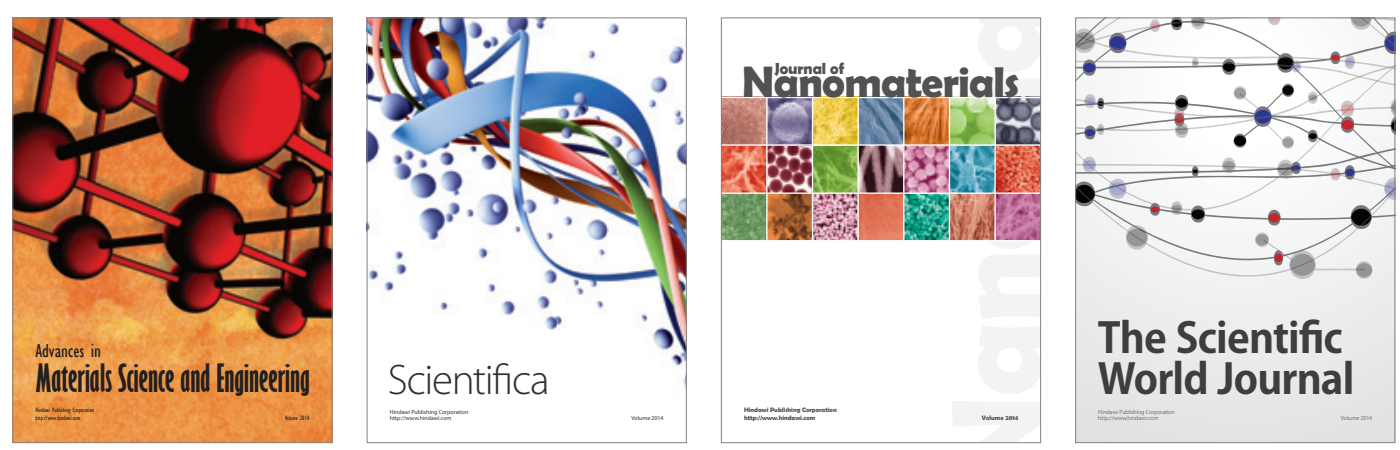

\section{The Scientific World Journal}
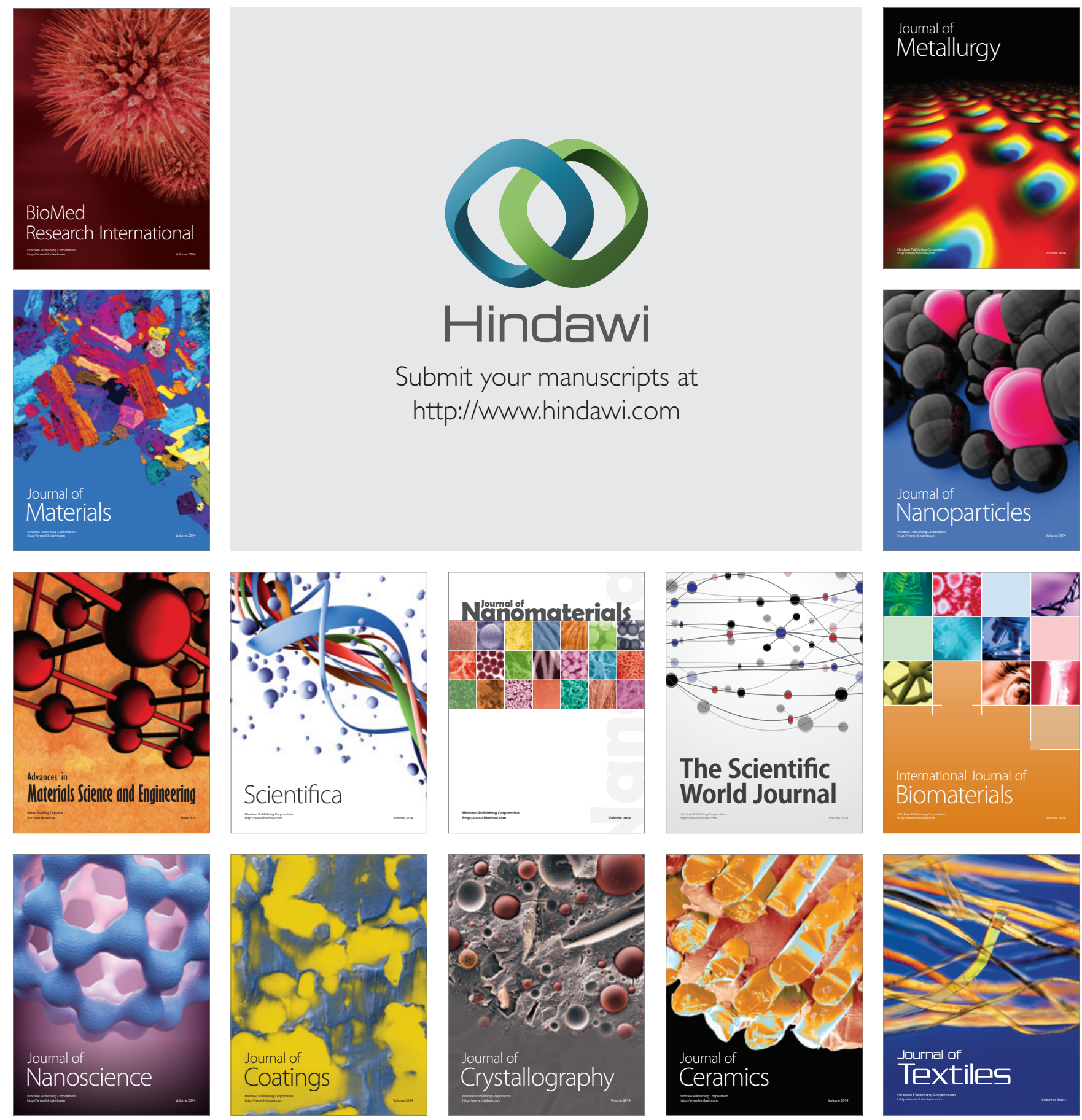